\title{
APROXIMACIÓN TEÓRICA AL CONCEPTO DE ESTADO: DISTINCIONES EN TORNO A HELLER, JELLINEK Y CARRÉ DE MALBERG ${ }^{1}$ Theoretical Approach to State Concept: Distinctions around Heller, Jellinek and Carré de Malberg
}

\section{Abraham Zamir Bechara Llanos ${ }^{2}$}

Fecha de recepción: 10 de febrero de 2015

Fecha de aceptación: 12 de mayo de 2015

SUMARIO: 1. Introducción; 2. Objeto y método en torno al concepto de Estado; 3. Antecedentes y concepto de la teoría del Estado; 4. Estructura del Estado; 5. Potestades y funciones del Estado; 6. Conclusiones; 7. Referencias bibliográficas.

\footnotetext{
${ }^{1}$ El presente artículo es resultado parcial de la investigación titulada: De los teóricos del Estado de derecho a los teóricos del Estado constitucional, distinciones conceptuales y analíticas en clave constitucional. Circunscrito al Grupo de Investigación Teoría Jurídica y Derechos Fundamentales Phronesis. Categoría D Colciencias. Línea de Investigación: Derechos Fundamentales.

${ }^{2}$ Abogado y Especialista en Derecho Constitucional, Universidad Libre. Maestrante en Derecho, Universidad del Norte. Docente de Filosofía del Derecho, Universidad de Cartagena. Docente de Metodología de la Investigación, Corporación Universitaria Rafael Núñez.
} 


\section{COMO SE CITA ESTE ARTÍCULO (APA 6)}

Bechara Llanos, Abraham Zamir (2015). Aproximación teórica al concepto de estado:Distinciones en torno a Heller, Jellinek y Carré De Malberg. Revista Jurídica Mario Alario D’Filippo, VII (14), pág 72-84.

\section{RESUMEN}

El trabajo realiza una aproximación analítica al concepto de Estado, estudiando a tres de los autores más representativos, y estableciendo conexiones teóricas entre ellos. El autor del artículo considera que pensar un estudio y análisis de la teoría del Estado sin el acompañamiento de la triada teórica propuesta, es negar y subvalorar los aportes invaluables que estos investigadores han hecho no solo a la ciencia del Estado en general, sino a la filosofía y a la ciencia política, a la teoría e historia del derecho del Estado, y en general a todo el cimiento base del derecho público-constitucional.

\section{PALABRAS CLAVE}

Teoría del Estado, ciencia política, historia del derecho, derecho público.

\section{ABSTRACT}

This paper makes an analytical approach to the concept of State, studying three of the most representative authors, and establishing theoretical connections between them. author considers that to think about a study and analysis of the theory of the State without the accompaniment of the proposed theoretical triad is to deny and undervalue the invaluable contributions of these researchers have made, not only to the science of the State in general, in the same way to philosophy and political science, to the theory and history of State law, and in general to the whole basis of public-constitutional law.

\section{KEYWORDS}

Theory of the State, political science, history of law, public law. 


\section{INTRODUCCIÓN}

El presente estudio pretende hacer una aproximación analítica al concepto de Estado, a través de tres de los autores más representativos: Hermann Heller, Georg Jellinek y Raymond Carré de Malberg. Los tópicos fundamentales que se tratarán en este texto -y que se dejarán a consideración del lector- son los siguientes: 1) objeto y método de la teoría del Estado; 2) antecedentes y concepto; 3) estructura del estado, y 4) potestades o funciones del Estado.

Como metodología de trabajo y para preservar la coherencia lógica en la construcción argumentativa de nuestro análisis, estableceremos conexiones teóricas de cada uno de los tres autores sobre los temas propuestos para nuestro desarrollo discursivo. En este sentido, el porqué de la importancia de un análisis a la teoría del Estado se funda en la necesidad de determinar las principales categorías jurídico-políticas que comprenden la misma noción de Estado y su trascendencia en el establecimiento, así como su institucionalización desde el punto de vista de los referentes teóricos, para el establecimiento de una mayor comprensión y aprendizaje de los mismos.

Nuestra hipótesis discursiva a lo largo del texto se centrará en abordar a cada uno de los autores, indagando por el acercamiento teórico desde sus aportes particulares y específicos sobre cada uno de los postulados que se mencionan. En dicho orden, pensar un estudio y análisis de la teoría del Estado sin el acompañamiento de esta triada teórica, es negar y subvalorar los aportes invaluables que han hecho no sólo a la ciencia del Estado en general, sino también a la filosofía y ciencia política, a la teoría e historia del derecho del Estado, y en general a todo el cimiento base del derecho público-constitucional en rasgos globales.

\section{OBJETO Y MÉTODO EN TORNO AL CONCEPTO DE ESTADO}

De conformidad con Heller, la teoría del Estado tiene como objeto investigar la específica realidad de la vida estatal que nos rodea, en la aspiración de comprender la estructura y función actuales del Estado, su devenir histórico y las tendencias de su evolución. En este orden, podemos extraer cuatro elementos analíticos sobre el objeto de la teoría del Estado.

Un primer elemento se centra en identificar la vida misma del Estado, que desde la unión de la ficción política del Estado como macroantropos según Platón, se debía erigir un hombre sobre los demás hombres ${ }^{3}$ como el más grande, el Estado por encima de todos

\footnotetext{
${ }^{3}$ Sobre este punto en particular, Carré de Malberg complementa la postura de Heller: "Es posible en efecto que los individuos que ha reunido el perseguimiento de un mismo fin, hayan contraído una asociación cuyo funcionamiento deba depender de las respectivas voluntades de cada uno de ellos. En este caso la voluntad común, destinada a realizar el fin común, no es sino la suma de las voluntades individuales, expresadas, bien sea por unanimidad, bien por simple mayoría de votos, por los propios miembros del grupo". (p. 47). Carré de Malberg, R. (1948). Teoría general del Estado. Trad. José Lion Depetré. Fondo de Cultura Económica. Colección Política y Derecho. Primera Edición en Español. México, D.F.
} 
los hombres, pero no sobre ellos. Como segundo elemento analítico, se configura el estudio de su estructura y funciones estatales, cuál debe ser la mejor estructuración estatal, así como las verdaderas funciones de un Estado en el reconocimiento pleno de los derechos y en el establecimiento eficaz de las garantías.

Como tercer elemento, el objeto del Estado se centra en estudiar su historia como componente transcendental, en aras de un desarrollo institucional ${ }^{4}$ del mismo. Y como cuarto elemento, se consolida el objeto de estudio de la evolución en torno a sus directrices institucionales. Siendo así, la relación de la teoría del Estado con el derecho y el mundo de lo jurídico se convierte en un componente transversal de su método, tal como lo destaca Heller (1998):

La actual teoría del Estado no puede aceptar aquella estrecha delimitación en su problemática que conocieron las dos últimas generaciones. Pues las teoría del Estado de esa época dejaron sin examinar gran parte de los más importantes problemas teóricos y prácticos, como los de la naturaleza, función y unidad del Estado, el de sus relaciones con la sociedad, la economía e, incluso, con el derecho, los del Estado de clase, de la opinión pública, de los partidos políticos, etc.; o bien, si se ocuparon de ellos, lo hicieron en medida insuficiente (p. 47).

Por lo cual respecto del objeto de la Teoría del Estado, luego de descartar por erróneas las concepciones científico-naturalistas del Estado, la de los espiritualistas y las de los idealistas, en palabras de Heller el objeto de la teoría del Estado es la investigación de la específica realidad de la vida estatal que nos rodea.

Según esta línea argumentativa, Jellinek va a proponer sobre el método de la teoría del Estado, una negación metodológica de antecedentes de forma en la literatura antigua, ya que según el autor alemán la construcción de un método para el estudio del Estado, debe realizarse teniendo en cuenta los parámetros modernos de medición de las ciencias sociales ${ }^{5}$, estableciendo una distinción conceptual sobre las ciencias naturales, básicas o exactas.

Guardando las proporciones con Kelsen, «Jellinek propone una pureza metodológica para el estudio de una verdadera teoría del Estado», principalmente al considerar que uno

\footnotetext{
4 "la única forma de continuar inscribiendo al Estado en la arena social implicaba concebirlo como expresión institucional de la correlación efectiva de las fuerzas políticas actuantes en un territorio dado. Lo propio del Estado, sin embargo, no era desintegrarse en una lucha irracional y nihilista por el poder, tal y como pretendían los veneradores de la violencia. Su valor sustantivo consistía en reconducir tal lucha, en tamizarla extrayendo su dimensión ética y constructiva, en transmutar la confrontación en una unidad de decisión amparada en principios compartidos de justicia". (p.161). Martín, S. (2015). Los fundamentos sociales, políticos y jurídicos del" soziale Rechtsstaat" Una relectura de Hermann Heller (1891-1933). Res Pública. Revista de Historia de las Ideas Políticas, Universidad Complutense de Madrid. (25), pp.151-175.

5 "De aquí ha nacido el que haya podido y pueda aun hoy suceder que, solo por el hecho de ser presentados con cierta firmeza, sean discutidos seriamente, como si se tratase de cuestiones científicas, casos que carecen de sentido. La afirmación de algo se convertía en hecho, la oscuridad pasaba por profundidad y la arbitraria especulación, por el más elevado conocimiento". (p. 73). Jellinek, G. (2000). Teoría general del Estado. Prólogo y trad. Fernando de los Ríos. Primera Ed. en español. Fondo de Cultura Económica. Colección Política y Derecho. México, D.F.
} 
de los problemas transcendentales del método de la doctrina estatal es, precisamente, que los estadistas y autores de la literatura de las ciencias del Estado encuentran graves dificultades en el estudio de los fenómenos fundamentales y las distinciones sutiles, en razón de no hacer deducciones e inferencias equívocas para no tomar imágenes y analogías por verdades reales.

Estos aspectos característicos del Estado dan lugar entonces a varios métodos de la teoría del Estado, a saber: el método científico o empírico-causal, el método filosófico, el método histórico y el método jurídico. Por ejemplo, dice en su obra que toda investigación que se haga hoy sobre los fenómenos fundamentales del Estado, debe comenzar por fijar los principios metodológicos, partiendo de los resultados de la nueva teoría del conocimiento y de las investigaciones realizadas en lógica; pues dice que solo así se poseerá un instrumento seguro, tanto para orientarse con un punto de vista crítico por la literatura antigua como para dar sustantividad y hacer fructifera la investigación. Entonces Jellinek traza en su obra, según sus mismas palabras, apenas lo que son las líneas principales de su método de estudio de la teoría del Estado.

En este sentido, la procura teórico-política de Jellinek se va a dirigir a la reivindicación de una teoría general del Estado en clave desde las ciencias sociales; además identifica, como problema subyacente, la «ruptura dialógica» entre la ciencia del Estado 6 con el propio origen de la ciencia social. A todo esto se suma, en palabras de Jellinek, la cuestión esencial de la falta de sistematización de la teoría, doctrina y dogmática del Estado:

Una lógica de las ciencias sociales, sistemática, comprensiva de todas las cuestiones difíciles, algo análogo a lo que se ha hecho con éxito para las ciencias naturales, apenas si se ha comenzado a trabajar, y aun lo poco que se ha hecho se refiere principalmente a la investigación de la historia, de la economía política, de la sociología, de la estadística; pero propiamente sobre la doctrina del Estado se encuentran muy pocas indicaciones (Jellinek, 2000. p. 73).

Georg Jellinek propone un «método histórico para la construcción de una verdadera teoría del Estado». Su cometido es identificar los principales acontecimientos históricos que tienen grandes consecuencias para el estudio de una ciencia estatal. En este sentido valida su tesis, de conformidad con la cual resulta totalmente necesaria la aplicación del método histórico para estudiar el universo estatal, si se quiere una comprensión científica de las instituciones, categorías o fenómenos que se van a estudiar. "Es ya hoy casi un lugar común el afirmar que para comprender científicamente una institución es un supuesto necesario el tener conocimiento de su historia. La escuela histórica del derecho fue quien hizo primeramente esta afirmación y quien la desarrollo" (2000, p. 85). Para conseguir este fin, el autor alemán recurre a establecer una distinción conceptual

\footnotetext{
${ }_{6}$ "Si el Estado es una unidad que actúa en la realidad histórico social, no podemos esquivar el examen, si quiera sea somero, de la difícil cuestión referente al carácter de esta realidad social. El punto de partida ha de ser aquí la fundamental e inconmovible afirmación de que la realidad social es efectividad humana, es realidad efectuada por el hombre". (p. 101). Jellinek, G. (2000).Teoría general del Estado. Segunda Edición en Español. Fondo de Cultura Económica. México, D.F.
} 
entre lo que significa y se entiende como cambio, por un lado, y lo que se percibe como evolución, por otro, identificando que no todo cambio histórico conlleva necesariamente a una evolución positiva del mismo, en función de un deber ser en el Estado. Jellinek distingue siete diferencias y conceptualizaciones teóricas en torno a la cuestión de que el cambio siempre es una muestra de la evolución del Estado:

Las instituciones cambian sin cesar, pero no todo cambio es una evolución; evolución existe solo en aquel cambio que conduce de lo más sencillo a lo más complicado. Cuando una institución se hace más amplia, más permanente o un fenómeno más intenso, cuando se acentúa su variedad, su poder director o su conformidad, entonces se dice que evoluciona. Las instituciones jurídicas cambian frecuentemente sin evolucionar y hasta, por el contrario, retroceden (Jellinek, 2000, p. 86).

De conformidad con lo anterior, Jellinek presenta varios ejemplos que podrían tomarse como iushistóricos, centrándose en la distinción cambio-evolución. Indica, en primer lugar, el origen de la «institución de los juicios por jurados» o jurados de conciencia, asimilados más al modelo del derecho anglosajón o del common law. En estos juicios quien toma la decisión no es el tercero imparcial y ajeno a las partes en conflicto, representado en la figura del juez. En los llamados juicios por jurados, los terceros de manera colectiva son los que toman la decisión a conciencia, es decir de conformidad con lo que "los jurados" logran abstraer de la realidad en los hechos, configurándose una realidad fáctica mas no una realidad jurídica o tomada de pleno derecho. Jellinek indica que esta institución nace en el derecho normando, y que luego es tomada por Inglaterra en el siglo XVI; finalmente, el juicio por jurados es transformado o reconstruido en Francia, destacando que ese cambio genera una transformación de la institución, no estableciendo una verdadera evolución. Las razones por las cuales Jellinek expresa que "no se dio la evolución de la institución», fueron que su adaptación al modelo del derecho del Estado francés no permitió el progreso de la misma, debido a que los jurados establecían su consideración con base eminente en lo fáctico, y el nacimiento de la institución en Normandía se concibió totalmente de forma opuesta, en virtud de que los jurados decidían sobre las pruebas en los procesos o litigios a los que eran llamados para actuar como tales.

Bajo esta línea argumentativa, Jellinek quiere destacar que la evolución o modificación de las instituciones sociales, políticas y jurídicas va a estar dado por los fines con los cuales estas fueron pensadas y construidas, y esos mismos fines puestos en contexto de lo presente y del ahora. Muchos de esos fines, pensados en épocas en las que el Estado se regía irrestrictamente por los postulados de la Monarquía, son totalmente distintos a los fines de una teoría del Estado construida en tiempos de una república, o aun en la misma monarquía constitucional o parlamentaria, según el modelo español y el de la monarquía parlamentaria en el Reino Unido. La modificación en los fines de las instituciones sociales es un fenómeno del más alto valor: 
Cuanta más vieja es una institución más probable es que no se hayan conservado sus fines primitivos o al menos que no se hayan mantenido en su forma inicial. De la naturaleza misma de las cosas depende que tratemos de modificar las instituciones políticas (Jellinek, 2000, p. 87).

Es así que cobra un papel muy importante el método histórico para la construcción y conformación de un objeto y método de la teoría del Estado. Jellinek destaca que no es necesario tener un conocimiento pleno o suficiente de todos los elementos históricos que conforman un antecedente de la institución, fenómeno o categoría que se esté investigando, al tiempo que enfatiza en este punto de su teoría del Estado que lo importante es el establecimiento del origen de los fines de la institución, no siendo relevados todos sus componentes históricos:

El saber en qué consiste la naturaleza esencial de la modificación de los fines de las instituciones sociales es decisivo, siempre que se trate de conocer en qué medida y de qué modo ha de hacerse la investigación histórica. Enseña esta primeramente que para comprender la esencia de un fenómeno actual no es necesario conocer todo su pasado, sino que es suficiente conocer desde aquel momento en que aparecen sus fines actuales, que es donde empieza su evolución (...) (Jellinek, 2000, p. 88).

\section{ANTECEDENTES Y CONCEPTO DE LA TEORÍA DEL ESTADO}

Sobre la teoría y estructura del Estado, Jellinek propone una configuración desde una asimilación objetiva del sentido estatal, esta no puede estar alejada de la realidad del mundo por fuera de las subjetividades individuales o del mismo hombre en sentido particular, el profesor alemán infiere, que si bien es importante la estructura objetiva del Estado, esta no puede ser exclusiva a la lectura de la ciencia estatal, ya que la plenitud del mundo subjetivo del hombre frente a la visión del Estado se traslada hacia un conocimiento en sentido objetivo-subjetivo. "Un modo tal de considerar el Estado, exclusivamente desde su aspecto exterior, esto es, objetivo, como le habremos de llamar, ofrece solamente una imagen pobre, e insuficiente científicamente del mismo" (Jellinek, 2000, p. 159).

Jellinek plantea que uno de los problemas acuciantes para la comprensión de una teoría general del Estado, y por ende de la consolidación de una ciencia estatal, es precisamente el atinente al concepto y naturaleza del Estado.

Antes de pretender dar una solución al problema más importante y difícil de la doctrina del Estado, a saber, el conocimiento de su naturaleza, es preciso indagar los puntos de vista desde los cuales se puede alcanzar un conocimiento del Estado (2000, p.159).

Puntualmente en los antecedentes y concepto del Estado, el autor alemán desarrolla la transcendencia del entendido estatal a través de cuatro componentes o partes integrantes de su concepto. Estas constituyen un elemento exterior del Estado, en el que 
las instituciones políticas y jurídicas del mismo son reflejadas y vistas hacia afuera, hacia la sociedad que construye la realidad social política. Un elemento interior, en el que el corazón del Estado posiciona elementos de su haber y sentir psíquico y psicológico, lo que el Estado o sus instituciones piensan de ellos mismos; esto va a reflejar una mirada interna dentro del apartado del Estado. Como tercer elemento está el objetivo, que indica la forma en las instituciones, sean percibidas de adentro (elemento interno) o hacia afuera (elemento externo social) deben reconocerse dentro de los límites de lo demostrable, verificable o contrastable. Y como cuarto elemento, dentro de la formación del concepto de Estado, está el elemento subjetivo que conlleva no solo la mirada interna, sino la externa desde la sociedad, pero con la característica esencial que es determinado por inferencias, o bien por razones que no se han contrastado o demostrado en la realidad social, política y jurídica del propio Estado.

En razón a lo anterior, Jellinek busca consolidar un sub-tipo de investigación subjetivainterior para explicar el concepto de Estado aplicable a la realidad social, dentro de una metodología histórica en términos de generalidad para la ciencia y doctrina del Estado. Sobre cuáles son las consideraciones puntuales, en un estudio subjetivo-interno del acontecer estatal, Jellinek destaca la especial ruptura con las ciencias naturales, pues un científico del Estado es un científico social y humano dentro de la reivindicación de las ciencias sociales y humanas. El quehacer subjetivo interior del Estado posibilita, en este sentido, dos formas o maneras de estudio de la "constelación estatal". En una primera medida, el Estado como realidad social, y en segunda medida el Estado como realidad o concepción jurídica. Es de nuestra propuesta argumentativa, mostrar cuál es la concepción jurídica del Estado en palabras de Jellinek:

La concepción jurídica del Estado tiene como objeto el conocimiento de las normas jurídicas que determinan y sirven de pauta a las instituciones y funciones del mismo, así como de las relaciones de los hechos reales de la vida del estado con aquellos juicios normativos sobre los que se apoya el pensamiento jurídico. La concepción jurídica del Estado se propone, por consiguiente, completa la concepción social del mismo; pero no puede, en modo alguno, confundirse una con otra (2000, p.161).

\section{ESTRUCTURA DEL ESTADO}

En cuanto a la estructura del Estado, Jellinek concibe su configuración en el sentido unitario griego, en la polis. Es la polis un cuerpo representativo desde la unitariedad, y dicha unidad le permite su consolidación desde el pensamiento antiguo hasta nuestros días, al recalcar el profesor alemán que es pensado como el paradigma del Estado su origen en la antigua Grecia. "En este Estado tipo, toda vida política parte del centro y vuelve a él. Los miembros del estado son, por tanto, los individuos aislados o formando asociaciones de familia, cuya vida política coincide con la del Estado" (2000, p.552). 
Jellinek identifica que el problema del Estado unitario, desde la visión griega, presenta muchos problemas en la actualidad, principalmente frente a las actuaciones de los asociados o administrados frente al mismo Estado, como cumplimiento de la concepción de legalidad o jurídica base del mismo principio de legalidad estatal. Problemas de centralización excesiva, problemas como la imposibilidad para desarrollar los órdenes locales no centrales, o los atinentes a las relaciones de las autoridades locales con las autoridades centrales estatales, «proponiendo un tipo ideal de Estado donde se reconozca la autonomía administrativa» de sus entes en los órdenes jerárquicos representativos del aparato estatal:

La doctrina de la autonomía administrativa toma otro giro en la segunda mitad del siglo XIX. Las investigaciones fundamentales sobre la evolución histórica del Estado inglés, unido a la convicción que hubo de tenerse en Alemania, gracias a los trabajos de Gneist especialmente, de que existía una relación íntima entre el self-government y la constitución del parlamento, mostró que ni jurídica ni políticamente se puede comprender bien a este sin tener un conocimiento exacto de la naturaleza y del modo de actuar las instituciones locales. (Jellinek, 2000, p.556).

Siguiendo a Heller, la manera como se distribuye el poder del Estado determina la forma del mismo; tal distinción es vital para entender la estructura del Estado. Sobre esto, el profesor alemán destaca que existen dos formas esenciales de Estado en su sentido eminentemente filosófico-político: la democracia y la autocracia. La democracia, en palabras de Heller (1998), "es una estructura de poder construida de abajo a arriba; la autocracia organiza al Estado de arriba abajo. En la democracia rige el principio de la soberanía del pueblo, en la autocracia el principio de la soberanía del dominador" (p. 313).

Esta teorización puede ser representada con la siguiente gráfica:

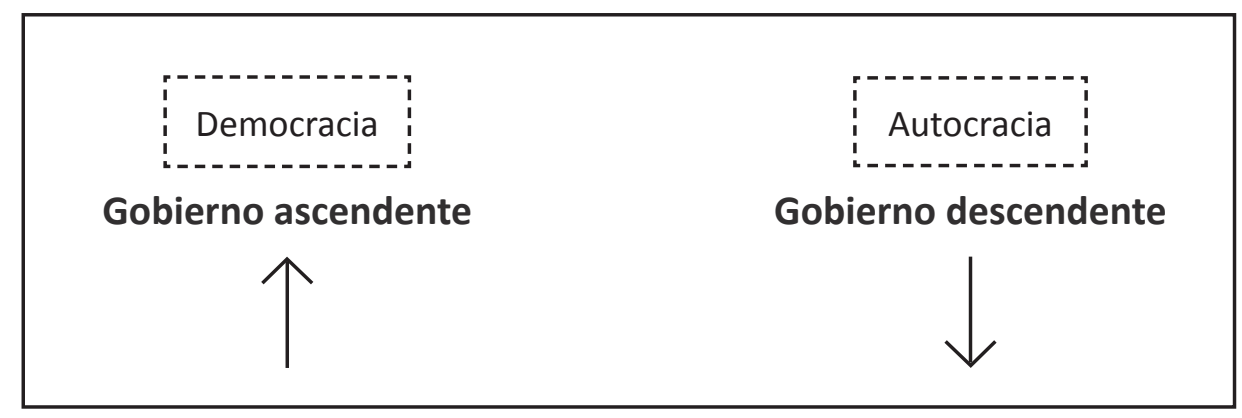

Grafica 1. Fuente propia.

\section{POTESTADES Y FUNCIONES DEL ESTADO}

Sobre las potestades y funciones del Estado, Carré de Malberg va a identificar la relación directa entre potestad y funciones, indicando que existen tres funciones principales al interior del Estado: Legislativa, Ejecutiva y Judicial, y que existen además tres potestades 
del Estado en igual sentido desde el poder Legislativo, Ejecutivo y Judicial. Entender las relaciones entre potestades y funciones discierne la actividad propia del Estado, así como las tres clases o cualidades de poderes que en términos del autor francés son:

Correspondientes a la triple naturaleza o fuerza especial de la potestad de que se hallan investidos respectivamente los órganos legislativos, ejecutivos y jurisdiccionales. A esta distinción de tres vías y potestades se refiere la clasificación de las funciones del Estado en legislativa, administrativa y jurisdiccional (Carré de Malberg, 1948, p. 80).

Las funciones estatales clásicas antes mencionadas encuentran eco en la división tripartita del poder público propuesta por Montesquieu, y han sido reivindicadas por el teórico francés Carré de Malberg. Aun en un Estado contemporáneo de derecho, se torna totalmente necesario el desarrollo primordial que se va a hacer a través de la función, el principio y la potestad de legalidad. Así las cosas, el principio de legalidad se erige como fundamento de la teoría de la función administrativa, en sede de su propia actividad. Desde esta perspectiva, el principio de legalidad se ubica como una directriz teóriconormativa, que orienta la función ejecutiva de la administración en cumplimiento de los postulados de la teoría del Estado.

En este sentido, el principio de legalidad encuentra una estrecha relación con la noción y las potestades del Estado, ya que el principio de legalidad como principio orientador de toda la función ejecutiva de administración estatal funge también como norma fundante del mismo concepto de Estado. Lo que resalta que la administración, en todo el ejercicio y despliegue de sus actos decisorios, debe velar y propender por el cumplimiento del principio de legalidad.

Finalmente, vemos en este aparte que el principio de legalidad ha permitido establecer unos elementos de dirección en la actividad propia del despliegue de la potestad administrativa, en palabras de Carré de Malberg, lo que ha desarrollado una fuerte noción por el respeto del principio de legalidad en su máxima expresión, como limite a las funciones públicas que ejerce la administración por intermedio de sus servidores y funcionarios públicos.

En cuanto a las funciones del Estado, para Heller estas han cambiado con el tiempo, pues dice este autor que en la Edad Media eran pocas las funciones que tenía el soberano, comparadas con las funciones que hoy existen en el Estado moderno; que en esa época, además, las funciones eran compartidas entre el soberano y otros estamentos privilegiados, como la Iglesia, la nobleza, los propietarios y los caballeros. Según Heller, entonces, la función del Estado es la de facilitar la organización interna para la división del trabajo y establecer las relaciones de intercambio e interdependencia.

Frente a las funciones del Estado, Heller (1998) identifica una nueva función del Estado en tiempos de modernidad: aquella que él denomina la función en la «organización 
autónoma de cooperación 7 social-territorial». A esta función le corresponde mirar las funciones del Estado más allá de su vínculo con su propio territorio; inclusive llegando a negar la idea de conformidad, con la cual la función del Estado no necesariamente es dada por una situación cultural y natural. Así las cosas, Heller es más vanguardista en el sentido de que construye los elementos de la ciencia del Estado moderno identificando, como rasgos característicos de ésta, la superación del Estado-Nación y la construcción de un verdadero Estado-Global.

Carré de Malberg, como primera medida, diferencia entre función y potestad estatal. En derecho público, se entiende por funciones estatales las diferentes actividades del Estado, en cuanto constituyen diferentes manifestaciones o diversos modos de ejercicio de la potestad estatal; para este autor, por el contrario, la potestad estatal es una y consiste en el poder que tiene el Estado de querer por sus órganos especiales por cuenta de la colectividad y de imponer su voluntad a los individuos.

Ahora, esas actividades del Estado que constituyen sus funciones, dice Carré de Malberg que se agrupan en tres: a) resguardar la seguridad de la nación respecto de las naciones extranjeras; b) asegurar en el interior, el orden y el derecho en las relaciones que entre sí mantienen los individuos, y c) desempeñar una misión cultural, en virtud de la cual ha de trabajar el Estado por sí mismo, o sea por cuantos medios especiales de que dispone, en el desarrollo de la prosperidad moral y material de la nación.

Pero diferencia además lo que es el objeto de la teoría jurídica de las funciones, para lo cual siguiendo una tradición antigua las concreta en tres actividades o funciones estatales, que son la legislación, la administración y la justicia, pero para determinar el alcance y objeto propio de cada una de ellas, se pregunta entonces ¿Cómo se ha llegado a distinguir una de otra la legislación, la administración, la justicia? Y como respuesta encuentra que se han dado muchas por diferentes escuelas, citando entre estas precisamente una de la cual George Jellinek es su mayor representante y que Malberg no comparte.

Según Carré de Malberg (1948), la potestad del Estado debe distinguirse de aquella fundamentación conceptual, que refleja la tesis personalísima de él, es decir, la idea que se erige desde la categoría Estado-persona. En este orden de ideas, el autor francés criticó expresamente la visión platónica del Estado, que era «defendida por el antropocentrismo platónico»:

El concepto del Estado-persona no basta para caracterizar al Estado. Además del Estado existe en efecto un gran número de formaciones humanas en las cuales la organización dada al grupo realiza la unidad personal de éste por encima de las personalidades individuales de sus miembros. Tal es el caso de las colectividades que corresponden a subdivisiones

\footnotetext{
7 "Esta necesidad de una solidaridad territorial para las cuestiones que de tiempo en tiempo se presenten en lo exterior, no es capaz, sin embargo, ni con mucho, de fundamentar la función de lo que desde el renacimiento conocemos como Estado. Hay que agregar un alto grado de división del trabajo social y, condicionada por ella, cierta permanencia y densidad de las relaciones de intercambio e interdependencia". (p. 259). HELLER, Hermann. Teoria del Estado.
} 
territoriales del Estado, como la provincia, el municipio, la colonia; tal es el caso también de muchas sociedades corporativas de derecho privado (Carré de Malberg, 1948, p. 80).

Esta potestad va a conformar un mandato que expresa un poder social, y «su función principal va ser la de asegurar su destino y funcionamiento", en otras palabras la propuesta en la ciencia del Estado en Carré de Malberg, está la posibilidad de una estabilidad estatal desde los cimientos populares, sociales, colectivos y ciudadanos. Quien entrega ese poder social al Estado, no es el ciudadano en sí mismo, ya que dicha concepción desvirtuaría su crítica antipersonalísima del Estado, por otorgarle un sentido unitario y colectivo de la potestad inherente por antonomasia del Estado, como lo es la soberanía. Es la soberanía la que le otorga ese rasgo distintivo, pero a su vez la Nación es la que le otorga la soberanía al Estado, como «autodeterminación democrática-agrupada en la nación» ${ }^{8}$.

Jellinek va a plantear sobre las funciones del Estado, un "abordaje histórico sobre la realidad de los acontecimientos» que marcaron la distribución en las funciones clásicas: legislativa, ejecutiva en cuanto al complemento en las sub-funciones de gobierno y administración, y la función judicial. Es contundente, en este aspecto, el profesor alemán al afirmar: "Todas las divisiones de las funciones del Estado, debidas a escritores de importancia, revelan que, al hacerla tuvieron presente al Estado concreto de su época y abstrajeron después de las actividades observadas, una doctrina general" (2000, p. 528).

De la división de las funciones del Estado dice, entonces, que solo tienen valor aquellas que penetran en la actividad del Estado y no se detienen en lo exterior, pero que ninguna división puede ofrecer una perfección lógica porque se trata es de comprender la vida y no una materia muerta, y todo lo vivo, aun cuando se dirija a la práctica, está a menudo privado de lógica. Señala que la primera división legítima es la que se basa en la doctrina de la división de poderes de Montesquieu, para quien hay en todo Estado tres clases de poderes: el legislativo, el ejecutivo y el judicial. Planteando la necesidad de separar las funciones en materiales y formales, es decir en distinguir las grandes direcciones de la actividad del Estado y las de determinados grupos de órganos, porque dice que la ingenua identificación entre actividad del órgano y función del estado, no puede dar solución a los más importantes problemas teóricos de la doctrina de las funciones. Considera entonces que las funciones materiales del Estado nacen de la relación entre la actividad del mismo y sus fines; y son entonces tres funciones materiales del Estado, a saber: la legislación, la jurisdicción y la administración.

La legislación, dice este autor, "establece una norma jurídica abstracta que regula una pluralidad de casos o un hecho individual"; la jurisdicción "fija en los casos individuales el derecho incierto o cuestionable o las situaciones o intereses jurídicos", y por último la administración "resuelve problemas concretos de acuerdo con las normas jurídicas,

\footnotetext{
8 "El concepto que pone en el Estado la potestad nacional no es, pues, una ficción teórica, sino que corresponde a realidades jurídicas. El estado, que en el curso de los estudios hechos con anterioridad se nos había presentado ya como el titular de la personalidad de la nación, se presenta ahora como siendo también el titular propio de la potestad nacional.” (pp. 80-81). Carré de Malberg, R. Teoría general del Estado.
} 
o dentro de los límites de ésta, valiéndose de medios que ante una investigación honda aparecen como un sistema complejo". Pero menciona Jellinek que, además de esas "funciones normales", existen otras actividades extraordinarias del Estado, tales como la guerra, en la cual lo que está en cuestión es la existencia misma del Estado.

\section{CONCLUSIONES}

La teoría del Estado se apoya en una construcción de un orden político jurídico, preestablecido para el cumplimiento de los desarrollos de los actos de voluntad de sus asociados como refleja la postura de Jellinek, decisorios de la potestad administrativa.

Sus posibles consecuencias, en relación con los asociados, están en la construcción como parte de la base de unos postulados, estrechamente relacionados con los principios y postulados del Estado, considerando que los alcances que persisten en la aplicación de una teoría del Estado en Hermann Heller, Georg Jellinek y el Francés Raymond Carré de Malberg, son precisamente por su gran observancia y pertinencia al interior de todo el andamiaje estatal moderno.

Como marco de posibilidades que el sistema estatal contemporáneo, le permite contar a los funcionarios de la administración, que finalmente son los que toman las decisiones expresadas en actos de voluntad Jellinek. En conclusión consideramos, que estas teorías del Estado perduran más allá de sus cultores, y se dan en la administración y en sus funcionarios, al aplicar y cumplimiento sus nociones, principios y directrices orientadores de la administración pública, como son que la función administrativa esté al servicio de los intereses generales y que los servidores públicos estén siempre dispuestos al servicio del Estado y la comunidad.

\section{REFERENCIAS}

Carré de Malberg, R. (1948). Teoría general del Estado. Traducción de José Lion Depetré. Fondo de Cultura Económica. Colección Política y Derecho. Primera Edición en Español. México, D.F.

Heller, H. (1998). Teoría del Estado. Edición y prólogo de Gerhart Niemeyer. Fondo de Cultura Económica. Segunda Edición en Español. México, D.F.

Jellinek, G. (2000).Teoría general del Estado. Prólogo y traducción de Fernando de los Ríos. Primera edición en español. Fondo de Cultura Económica. Colección Política y Derecho. Segunda Edición en Español. México, D.F.

Martín, S. (2015). Los fundamentos sociales, políticos y jurídicos del "soziale Rechtsstaat" Una relectura de Hermann Heller (1891-1933). Res Pública. Revista de Historia de las Ideas Políticas. Universidad Complutense de Madrid. (25), pp.151-175. 